\title{
Síntomas asociados al sufrimiento en pacientes con enfermedad renal crónica en hemodiálisis
}

\author{
Claudia Rodríguez-Ramírez ${ }^{1}$, Yadira Grau-Valdés², Jorge A. Grau-Ábalo ${ }^{3}$ \\ ${ }^{1}$ Servicio de Nefrología. Hospital Docente Clínico Quirúrgico "Doctor Salvador Allende". La Habana. Cuba \\ ${ }^{2}$ Facultad de Psicología de la Universidad de Málaga. España \\ ${ }^{3}$ Departamento de Psicología. Facultad de Ciencias Médicas de La Habana "Manuel Fajardo". Cuba
}

Como citar este artículo: Rodríguez-Ramírez C, Grau-Valdés Y, Grau-Ábalo JA. Síntomas asociados al sufrimiento en pacientes con enfermedad renal crónica en hemodiálisis. Enferm Nefrol. 2021 Jul-Sep;24(3):295-302

\section{Resumen}

Introducción: Los síntomas insuficientemente controlados pueden constituir un factor determinante 0 agravante del sufrimiento en los pacientes sometidos a hemodiálisis; sin embargo, escasean las investigaciones que aborden este tema.

Objetivos: El presente estudio tiene como objetivo describir el sufrimiento según la severidad de determinados síntomas en pacientes con enfermedad renal crónica en hemodiálisis.

Material y Método: El estudio es observacional descriptivo transversal, con algunas tareas de correlación en una muestra de 31 pacientes. Se emplearon la entrevista, el instrumento para detección de bienestar/malestar propuesto por Bayés y colaboradores y una serie de sub-escalas de la Escala de Evaluación de Síntomas de Edmonton/ESAS para identificar la presencia de síntomas (intensidad y frecuencia) que pudieran estar asociados al sufrimiento.

Resultados: El 87,1 \% del total mostró un bajo predominio de síntomas con severa intensidad asociados al sufrimiento en las últimas 24 horas, el 77,4\% bajo predominio en la semana anterior y el $61,3 \%$ un bajo

\section{Correspondencia:}

Claudia Rodríguez Ramírez.

crr@infomed.sld.cu predominio en un periodo de un mes. No se encontró asociación significativa entre el predominio de los síntomas con intensidad severa asociados al sufrimiento en 24 horas.

Conclusiones: En la mayoría de los sujetos predominó el bajo predominio de síntomas con severa intensidad asociados al sufrimiento; la magnitud de la sintomatología no constituyó un factor importante asociado al sufrimiento en estos pacientes.

PALABRAS CLAVE: sufrimiento; síntomas; enfermedad renal crónica; hemodiálisis.

\section{Symptoms associated to suffering among patients with End-Stage Renal Disease undergoing Hemodialysis}

Introduction: Insufficiently controlled symptoms could be a determining factor or aggravation of suffering in patients undergoing hemodialysis. However, research about this topic is not enough.

Objectives: This study aims to describe suffering according to severity of symptoms in patients with End-Stage Renal Disease undergoing hemodialysis.

Materials and Method: The study is transversal, descriptive and observational with some tasks of correla- 
tion in a sample of 31 patients. The assessment tools were; the interview, the instrument to detect wellbeing/ disturbance proposed by Bayés and collaborators and a series of subscales of the Edmonton Symptom Assessment Scale/ESAS to identify the intensity and frequency of symptoms that could be associated to suffering.

Results: $87.1 \%$ of the sample showed a low presence of symptoms with severe intensity associated to suffering in the last 24 hours, $77.4 \%$ showed low presence in the last week and $61.3 \%$ showed a low presence in a period of one month. No significant association was found between the presence of symptoms with severe intensity associated to suffering in $\mathbf{2 4}$ hours.

Conclusions: The majority of subjects were characterized by low presence of symptoms with severe intensity associated to that suffering. The magnitude of a set of symptoms did not result in an important factor associated to the suffering in these patients.

KEY WORDS: suffering; symptoms; chronic kindney disease; hemodialysis.

\section{Introducción}

Desde hace algunos años, se ha acrecentado la preocupación por los cambios en la salud de la población a nivel mundial debido a las enfermedades crónicas ${ }^{1}$. Los enfermos crónicos avanzados experimentan múltiples problemas que son consecuencias de la enfermedad, de los tratamientos médicos o de las complicaciones derivadas de los propios tratamientos. Estas consecuencias pueden ser determinantes 0 agravantes de sufrimiento e influir de muy variadas formas tanto en el paciente como en sus familiares más cercanos y en los miembros del equipo de salud ${ }^{2,3}$.

La conceptualización del "sufrimiento" ha sido reciente y polémica, así como su valoración y manejo en enfermos crónicos, familiares y personal de salud ${ }^{4}$. Inicialmente fue definido por Cassell ${ }^{5}$ como el malestar generado por la percepción (actual o no) de algo que amenaza la integridad o el bienestar de la persona. Se trata de una experiencia que abarca la totalidad de los aspectos que componen al ser humano y se manifiesta de manera individual, solo puede ser descrita por quien la vivencia.

Chapman y Gravin ${ }^{6}$ lo consideraron como un complejo estado afectivo, cognitivo y negativo que se caracteriza por la sensación de amenaza a la integridad, por un sentimiento de impotencia para hacerle frente a tal amena- za y por el agotamiento de los recursos personales que permitan enfrentarla. Muchos autores han contribuido a su diferenciación de los términos estrés, dolor y depresión y han destacado la importancia que tiene la percepción de amenaza en el sufrimiento y su singularidad como experiencia subjetiva individual ${ }^{7-10}$.

En realidad, el sufrimiento entra de lleno como categoría psicológica, con métodos de exploración, hace relativamente poco tiempo con los trabajos de Ramón Bayés y colaboradores ${ }^{11}$. Estos autores reiteran que una persona sufre cuando: a) experimenta o espera que le acontezca un malestar físico o psicosocial que valora como amenaza importante para su existencia o integridad física y/o psicosocial o para la de personas queridas, y, b) cuando cree que carece de recursos o se siente sin fuerzas para afrontar dicha amenaza. El sufrimiento aparece aquí como un constructo subjetivo susceptible de definirse operacionalmente, en el cual no solo participa la percepción de amenaza (síntomas, complicaciones, pronósticos inciertos, limitaciones funcionales, entre otros) sino también la carencia de recursos personales (afrontamientos, estilos atribucionales, disposiciones salutogénicas, sentidos de vida, esperanzas, entre otros).

Entre las enfermedades crónicas más frecuentes se encuentran las enfermedades renales. Estos pacientes en etapas avanzadas experimentan una elevada carga de síntomas relacionados con el fallo renal; aquellos que se hemodializan, se encuentran entre los grupos de pacientes crónicos más sintomáticos, vulnerables y afectados psicosocialmente $\mathrm{e}^{2,12-19}$.

Se considera que la carga de síntomas de la enfermedad renal crónica en estadios avanzados es similar a la de otras enfermedades crónicas en estas etapas ${ }^{20,21}$ y que los síntomas más frecuentes son: debilidad, astenia, dolor, prurito, estreñimiento, disnea, ansiedad, depresión, náuseas, trastornos del sueño, entre otros $22-24$.

Los síntomas insuficientemente controlados pueden constituir un importante determinante 0 agravante de sufrimiento $0^{9,11}$ y uno de los factores que más afecta la calidad de vida y la optimización de los tratamientos médicos ${ }^{15-17,19,22,25}$. Numerosas variables psicosociales y trastornos emocionales relacionados con el sufrimiento también intervienen en la aparición de síntomas somáticos o modulan la percepción que tienen los pacientes sobre los mismos ${ }^{16,26-30}$

La prevención y el alivio tanto de los síntomas como del sufrimiento constituyen prioridades para el cuidado 
paliativo en estos pacientes ${ }^{12,15,31-33}$. Sin embargo, son escasas las investigaciones que abordan la vinculación entre sintomatología y sufrimiento en pacientes que reciben hemodiálisis crónica. Así, el objetivo de este trabajo fue describir el sufrimiento según la severidad de determinados síntomas en pacientes con enfermedad renal crónica en hemodiálisis.

\section{Material y Método}

Estudio observacional descriptivo de corte transversal con algunas tareas de correlación entre las variables de síntomas y sufrimiento. La muestra era de 31 pacientes que reciben hemodiálisis crónica hospitalaria como tratamiento sustitutivo de la función renal en el Servicio de Hemodiálisis del Hospital "Doctor Salvador Allende" de La Habana en el segundo semestre del 2019, que desearan participar en el estudio y cuyo estado mental y físico les permitieran responder a las preguntas contenidas en los instrumentos.

Se emplearon los siguientes instrumentos evaluativos:

- Entrevista elaborada por los autores, para obtener datos sociodemográficos y clínicos de los pacientes y explorar con mayor énfasis el predominio de las vivencias de bienestar/malestar y su relación con síntomas.

- Instrumento propuesto por Bayés y colaboradores para la detección de sufrimiento y de algunos de sus determinantes (considerando al malestar emocional como su eje medular) mediante dos preguntas sencillas que pueden introducirse en el contexto clínico habitual sin involucrar problemas éticos: 1) ¿Cómo se le hizo el tiempo en el día de ayer: corto, largo o Ud. que diría? 2) ¿Por qué?

Se basa en la percepción del tiempo (subjetivo versus objetivo), que ha sido usada como un marcador del bienestar o del malestar. Si el paciente refiere percibir un paso del tiempo "largo", es indicativo de malestar/sufrimiento, mientras que la percepción del paso del tiempo "corto" se corresponde a la presencia de bienestar/no sufrimiento ${ }^{34}$. Si la respuesta que proporciona el paciente a la segunda pregunta no se relaciona con ningún síntoma, pudiera ser que los síntomas no constituyen un determinante 0 agravante de sufrimiento en el momento concreto en que se formuló la pregunta. Cuando la respuesta se relacione con algún síntoma, será necesario utilizar instrumentos diagnósticos más precisos.

Este instrumento ha sido probado en repetidas ocasiones en pacientes oncológicos españoles y con
SIDA y también en estudiantes de un Instituto de Educación Física en Cataluña, con altas correlaciones entre el malestar y el tiempo subjetivo alargado y entre el bienestar con la percepción de tiempo acortado, independientemente de variables sociodemográficas y clínicas ${ }^{34}$.

Se ha empleado con éxito en pacientes oncológicos cubanos en diferentes estadíos de la enfermedad, con similares resultados 3,35 y en una investigación con pacientes renales bajo tratamiento de hemodiálisis iterada (HD), demostrándose efectividad diagnóstica de $94 \%{ }^{14}$.

- Sub-escalas de la Escala de Evaluación de Síntomas de Edmonton/ESAS de Bruera y colaboradores ${ }^{36}$, modificada por los autores para la identificación de intensidad y frecuencia de síntomas que pudieran ocasionar malestar. Teniendo en cuenta que se trata de sub-escalas de auto-reporte (de naturaleza ordinal y que se interpretan como escalas numéricas), cuya validez y sensibilidad ha sido probada y no varía con adaptaciones ni versiones; a los fines del presente estudio se agregaron a la escala original otras sub-escalas de síntomas frecuentes en pacientes renales: debilidad, prurito, calambres musculares, estreñimiento y dificultades sexuales.

Se consideró estimar tres puntuaciones en diferentes lapsos de tiempo para disponer de una valoración sostenida de síntomas que pudieran asociarse al sufrimiento: en las últimas 24 horas, durante la semana anterior y en el mes precedente a la exploración.

Este estudio fue aprobado por el Comité Científico y de Ética de la Investigación del Hospital "Doctor Salvador Allende". La aplicación de los instrumentos se realizó de forma individual en una sola sesión y durante el proceder dialítico, con duración máxima de 45 minutos. Se intentó prevenir agotamiento o malestar aplicando los instrumentos en momentos en que se sentían con relativo bienestar. Se solicitó el consentimiento informado de los pacientes por escrito antes de iniciar el proceso evaluativo.

Los resultados fueron analizados cuantitativamente empleando el paquete estadístico SPSS versión 21 siempre que fuese posible y de forma cualitativa, mediante la triangulación de la información obtenida en la entrevista, en el Instrumento propuesto por Bayés y colaboradores y en las sub-escalas de síntomas. Con vista a establecer asociaciones entre la presencia de malestar y determinados síntomas, se empleó la prueba 
Ji cuadrado de independencia, dado el tipo de variables incluidas en el análisis. En el caso de variables ordinales, se utilizaron las pruebas Ridit Analysis o de Bartholomew según el número de categorías. Se fijaron niveles de significación de $p<0,05$ y se construyeron tablas con vista a representar de forma resumida la información encontrada.

\section{Resultados}

La muestra quedó constituida por 31 pacientes con una media de edad de $60 \pm 11,6$ años. Del total, el $71,0 \%$ fueron hombres, el 35,5\% finalizó el nivel de escolaridad medio superior, el 25,8\% refirió tener al menos un hijo, el $51,6 \%$ comentó tener una pareja estable y el $58,1 \%$ estaba jubilado. En cuanto a las variables clínicas, el 58,1\% del total tenía una comorbilidad de 1 a 4 enfermedades, el 74,2\% tenía como acceso vascular la FAV y el $64,5 \%$ no recibió preparación para la diálisis. Las medias del tiempo del diagnóstico de la enfermedad y del tiempo en hemodiálisis fueron de $74,2 \pm 36,9$ meses, y de $81,3 \pm 30,2$ meses. El $77,4 \%$ estaba diagnosticado como no apto para el trasplante renal por diferentes razones: por la realización de los estudios pertinentes para el trasplante renal, por la estabilización médica tras algún problema de salud situacional, por la edad o la comorbilidad asociada, entre otras.

Según la entrevista, el bienestar se asoció, fundamentalmente, al no padecimiento de síntomas en magnitud severa. En el instrumento propuesto por Bayés y colaboradores, en la primera pregunta contestaron: "lento (largo)" el 6,5\% de los casos, "rápido (corto)" el $19,3 \%$ y con "otra denominación" el 74,2\% del total. Estos resultados obligaron a indagar, mediante un análisis de contenido, cuántos de aquellos sujetos que respondieron con "otra denominación" tenían en sus respuestas una connotación positiva o negativa. Para este análisis se conformaron dos grupos de formulaciones:

1. De connotación positiva con respuestas de:

$$
\begin{aligned}
& \text { " "Bien", "Normal" } \\
& \text { " "Rapidísimo" } \\
& \text { - "Demasiado rápido" } \\
& \text { - "Bastante corto" } \\
& \text { " "Bien el día" } \\
& \text { " "Día que más trabajé" }
\end{aligned}
$$

2. De connotación negativa (relacionadas con el aburrimiento, con la rotura de la planta de agua de la unidad de diálisis, con la espera del turno de diálisis o el tener que asumir todas las labores domésticas) con respuestas de:

- "Larguísimo los días"

- "Muy violento" "Un poco lento", "algo lento"

Para el análisis de la segunda pregunta: "¿Por qué?", las respuestas se categorizaron en ocho grupos de formulaciones que son presentados en orden de mayor a menor frecuencia (los cuatro primeros lugares tuvieron una connotación positiva, el resto se referían a determinantes de malestar):

1. Distracciones, recreaciones (viendo TV, películas, novelas)

2. Interactuando con la familia y amistades, en visitas

3. Ocupado en quehaceres domésticos, trabajando, resolviendo asuntos personales.

4. Por sentirse bien

5. Por sentirme solo, aburrido o mal

6. Rotura de la planta de agua, lo cual atrasa las sesiones de HD

7. Espera de la HD al otro día para sentirse mejor

8. Tener que hacer todos los quehaceres domésticos

En la correlación entre el tiempo subjetivo y las expresiones de bienestar/malestar (ver tabla 1) se obtuvo que el $96 \%$ de los pacientes tenían explicaciones de bienestar a partir de una respuesta de "rápido (corto)" u "otra denominación" positiva, y que el $100 \%$ identificaban el malestar con respuestas de "lento (largo)" u "otra denominación" en sentido negativo.

\begin{tabular}{|c|c|c|c|c|c|}
\hline \multicolumn{5}{|c|}{ Instrumento para la Detección del Sufrimiento (Bayés y cols.) } & \multirow{3}{*}{ Total } \\
\hline \multirow{2}{*}{ Respuestas } & \multicolumn{2}{|c|}{$\begin{array}{l}\text { Explicaciones } \\
\text { de malestar }\end{array}$} & \multicolumn{2}{|c|}{$\begin{array}{l}\text { Explicaciones } \\
\text { de bienestar }\end{array}$} & \\
\hline & Casos & $\%$ & Casos & $\%$ & \\
\hline Lento u otra denominación negativa & 6 & 100 & 0 & 0 & 6 \\
\hline Rápido u otra denominación positiva & 1 & 14,3 & 24 & 96 & 25 \\
\hline Total & 7 & 100 & 24 & 100 & 31 \\
\hline
\end{tabular}

La distribución de pacientes según el predominio de los síntomas con intensidad severa asociados al sufrimiento en un periodo de 24 horas según las sub-esca-

Tabla 1. Distribución de pacientes según el tiempo subjetivo con explicaciones de malestar/bienestar. 
Tabla 2. Distribución de pacientes según el predominio de los síntomas con intensidad severa asociados al sufrimiento en un periodo de 24 horas, 1 semana y 1 mes.

\begin{tabular}{|c|c|c|c|c|c|c|}
\hline \multirow{2}{*}{$\begin{array}{l}\text { Predominio de los síntomas con severa intensidad asociados al } \\
\text { sufrimiento }\end{array}$} & \multicolumn{2}{|c|}{$24 \mathrm{~h}$} & \multicolumn{2}{|c|}{1 semana } & \multicolumn{2}{|c|}{1 mes } \\
\hline & casos & $\%$ & casos & $\%$ & casos & $\%$ \\
\hline Sin predominio (ningún síntoma con intensidad severa: 7-10) & 6 & 19,4 & 4 & 12,19 & 4 & 12,19 \\
\hline Bajo (1-3 síntomas con intensidad severa: 7-10) & 21 & 67,7 & 24 & 77,4 & 19 & 61,3 \\
\hline Moderado (4-7 síntomas con intensidad severa: 7-10) & 4 & 12,9 & 3 & 9,7 & 8 & 25,8 \\
\hline Alto (8-13 síntomas con intensidad severa: $7-10$ ) & 0 & 0 & 0 & 0 & 0 & 0 \\
\hline Total & 31 & 100 & 31 & 100 & 31 & 100 \\
\hline
\end{tabular}

las de síntomas (ver tabla 2), mostró que el 67,7 \% de los pacientes tuvo un bajo predominio de síntomas con severa intensidad en las últimas 24 horas. En cuanto a la determinación final de la presencia o no de sufrimiento según el predominio de los síntomas con intensidad severa o de la presencia de 2 de los siguientes síntomas en intensidad severa: debilidad, astenia, dolor, prurito, estreñimiento, disnea, ansiedad, depresión, náuseas, cambios en el patrón de sueño en el lapso de 24 horas (ver tabla 3); el 87,1\% tuvo un bajo predominio de síntomas de severa intensidad asociados al sufrimiento. El $77,4 \%$ presentó un bajo predominio de síntomas de severa intensidad asociados al sufrimiento en la semana precedente y el $61,3 \%$ mostró un bajo predominio de tales síntomas en el mes anterior (ver tabla 2).

Los síntomas con intensidad severa que se identificaron en los tres lapsos de tiempo (con independencia del nivel de predominio) fueron: "dificultades para dormir", "prurito", "malestar", "falta de aire", "cansancio",
Tabla 3. Distribución de pacientes según presencia o ausencia de sufrimiento según el malestar asociado al predominio de intensidad severa en síntomas en 24 horas.

\begin{tabular}{l|c|c}
\hline $\begin{array}{l}\text { Presencia de sufrimiento según el } \\
\text { predominio de síntomas con severa } \\
\text { intensidad en el ESAS } \\
\text { (24 horas) }\end{array}$ & Casos & $\%$ \\
\hline Presencia de sufrimiento & 4 & 12,9 \\
\hline Ausencia de sufrimiento & 27 & 87,1 \\
\hline Total & 31 & 100 \\
\hline
\end{tabular}

"somnolencia", "calambres", "hipotensión", "ansiedad" y "dificultades sexuales". No se encontró asociación significativa entre el predominio de los síntomas con intensidad severa asociados al sufrimiento en 24 horas con la prueba de probabilidad exacta de Fisher $(1,000)$ (ver tabla 4$)$.

Tabla 4. Distribución de pacientes según la contrastación entre el predominio del malestar según el ESAS 24 horas y el malestar/ bienestar global.

\begin{tabular}{|c|c|c|c|c|c|c|c|}
\hline \multirow{4}{*}{$\begin{array}{l}\text { Síntomas asociados a la vivencia de malestar } \\
\text { de acuerdo al predominio de su severa } \\
\text { intensidad }\end{array}$} & & \multicolumn{2}{|c|}{ Malestar } & \multicolumn{2}{|c|}{ Bienestar } & \multicolumn{2}{|c|}{ Total } \\
\hline & & casos & $\%$ & casos & $\%$ & casos & número \\
\hline & Malestar & 1 & 8,3 & 2 & 10,5 & 3 & 9,7 \\
\hline & Bienestar & 11 & 91,7 & 17 & 89,5 & 28 & 90,3 \\
\hline Total & & 12 & 100 & 19 & 100 & 31 & 100 \\
\hline
\end{tabular}

Fisher 1,000 


\section{Discusión}

Los resultados obtenidos en el instrumento de detección del sufrimiento propuesto por Bayés y colaboradores fueron similares a los reportados en pacientes oncológicos cubanos en investigaciones precedentes, en el sentido del predominio de respuestas con "otra denominación", a pesar de que la diversidad de categorías de respuesta fue más reducida que en investigaciones pre$v$ vias $^{35}$. Esto pudiera deberse a pequeñas diferencias socioculturales en relación con la expresión de vivencias, además de diferencias asociadas a las enfermedades de base de los pacientes incluidos en los estudios.

Este instrumento demostró tener una alta potencia-eficiencia diagnóstica (96\%) para la identificación del bienestar a partir de una respuesta de "rápido (corto)" u "otra denominación positiva" y en un $100 \%$ para la identificación del malestar con una respuesta de "lento (largo)" u "otra denominación en sentido negativo". Estos resultados encontrados han sido superiores a los reportados por estudios españoles y cubanos anteriores $^{3,11,34,35}$.

Los resultados indicaron que en la mayoría de los sujetos estudiados predominó el bajo predominio de síntomas con severa intensidad asociados al sufrimiento, lo que indicó que la sintomatología en magnitud severa no constituyó, en el momento de la exploración, un determinante importante asociado al sufrimiento en estos pacientes.

La diversidad de síntomas identificados en los tres lapsos de tiempo concuerda con lo planteado por la literatura científica: este grupo de pacientes experimenta una gran variedad de síntomas cambiantes relacionados con el fallo renal y se encuentra entre los grupos de pacientes con enfermedades crónicas más sintomáticas $^{12,15,17-19}$.

A pesar de que el equipo profesional, junto con los familiares, trabajan en base al control y alivio del dolor y demás síntomas, un aspecto que pudiera haber influido en los resultados obtenidos, y que sugiere profundización en investigaciones posteriores con muestras mayores lo constituyen, en primer lugar, la confirmación de que no es la magnitud severa de los síntomas el factor que más contribuye al sufrimiento global (como se plantea en la conceptualización del sufrimiento), y en segundo lugar, las consideraciones éticas relacionadas con el momento apropiado para la evaluación psicológica. Debe recordarse que los instrumentos fueron aplicados cuando los pacientes ofrecían su pleno consentimiento y disposición para ello, lo que hace suponer que haya sido en momentos de relativo bienestar.

A partir de los resultados encontrados podemos concluir que la mayoría de los sujetos estudiados tenía un bajo predominio de síntomas con severa intensidad asociados al sufrimiento, lo que indicó que la magnitud severa de los síntomas no constituyó un determinante importante asociado al sufrimiento en estos pacientes.

Conflicto de interés: No se declaran conflictos de interés por los autores.

Financiación: La presente investigación no ha recibido ayudas específicas provenientes de agencias del sector público, sector comercial o entidades sin ánimo de lucro; se ha insertado en el trabajo asistencial cotidiano de la primera autora en la unidad de hemodiálisis donde trabaja.

Recepción: 10-06-21

Aceptación: 25-07-21

Publicación: 30-09-21

\section{Bibliografía}

1. OMS/Organización Mundial de la Salud. Prevención y control de las enfermedades no transmisibles: aplicación de la estrategia mundial. Resolución de la 122 Reunión del Consejo Ejecutivo de la OMS, enero 2008.

2. Grau Valdés $Y$, Rodríguez Umpierre R, Martínez Rodríguez $\mathrm{L}$. Sentido personal del rol y estrategias de afrontamiento en cuidadores de pacientes en hemodiálisis. International Journal of Developmental and Educational Psychology INFAD. 2019;1:269-82.

3. Grau J, Rodríguez C, Chacón M. A avaliação do sofrimento no fim da vida: uma revisão da perspectiva do psicodiagnóstico. En: VA Angerami (comp) Atuação do psicólogo no contexto da saúde. Belo Horizonte: Artesa Editora. 2019;453-80. 
4. Grau J.Vivir con una enfermedad crónica. Guadalajara: UNIDAPSA. 2017.

5. Cassell EJ. Recognizing suffering. Hastings Cent Rep. 1999;21:24-8.

6. Chapman CR, Gravin J. Suffering: the contributions of persistent pain. Lancet. 1999;353:2233-7.

7. Fordyce WE. Pain and suffering: what is the unit? Quality of Life Research. 1994;3(1):551-6.

8. Loeser JD, Melzack R. Pain: an overview. Lancet. 1999;353:1607-9.

9. Gómez Sancho M, Grau J. Dolor y sufrimiento al final de la vida. Madrid: Arán, 2006.

10. Krikorian A, Limonero JT, Maté J. Suffering and distress and the end of life. Psychooncology. 2012;21(8):799808.

11. Bayés R. Psicología del sufrimiento y de la muerte. Barcelona: Martínez Roca, 2001.

12. Chambers EJ, Germain M, Brown E. Supportive care for the renal patient. Oxford University Press, 2004.

13. Axelsson L, Randers I, Jacobson S, Klang B. Living with hemodialysis when nearing end of life. Scandinavian Journal of caring sciences 2012;26(1),45-52.

14. Rodríguez C. Necesidades espirituales en pacientes que se hemodializan en el Hospital "Salvador Allende". Tesina del Diplomado Nacional en Cuidados Paliativos, Universidad de Ciencias Médicas de La Habana, 2014.

15. Gutiérrez D, Leiva JP, Sánchez R, Gómez R. Prevalencia y evaluación de síntomas en enfermedad renal crónica avanzada. Enferm Nefrol 2015,18(3):228-36.

16. Davison SN, Levin A, Moss AH, Brown EA, Brennan F, et al. Executive summary of the KDIGO Controversies Conference on Supportive Care in Chronic Kidney Disease: Developing a roadmap to improving quality care. Kidney Int 2015;88(3):447-59.

17. Ortega $E$, Sánchez $C$, Serrano R, Lamana $A$, Santos B, Sanz M, Saharaui Y, Sánchez, J.A. Prevalencia de síntomas en enfermedad renal crónica avanzada. Nefrología 2018;38(5):558-72.
18. Lanatta V, Quiñones C, Paredes JL, Sarabia S, León C. Alta frecuencia de sintomatología depresiva en cuidadores de pacientes pediátricos en diálisis peritoneal y hemodiálisis en un hospital público de Lima. Rev Neuropsiquiatr 2019;82(3):202-20.

19. Hernández-Zambrano SM, Torres $M L$, Barrero SS, Saldaña IJ, Sotelo KD, Carrillo AJ, Rodríguez KA. Necesidades de cuidado paliativo en hemodiálisis percibidas por pacientes, cuidadores principales informales y profesionales de enfermería. Enferm Nefrol 2019;22(2):141-9.

20. Solano JP, Gomes B, Higginson IJ. A comparison of symptom prevalence in far advanced cancer, AIDS, heart disease, chronic obstructive pulmonary disease and renal disease. Journal of pain and symptom management 2006;31(1):58-69.

21. Janssen DJA, Spruit MA, Wouters EFM, Schols JMGA. Daily symptom burden in end-stage chronic organ failure: a systematic review. Palliat Med 2008;22(8):93848.

22. Murtagh FEM, Addington-Hall J, Higginson IJ. The prevalence of symptoms in end- stage renal disease: a systematic review. Advances in chronic kidney disease 2007;14(1):82-99.

23. $0^{\prime}$ Connor NR, Kumar P. Conservative management of end-stage renal disease without dialysis: a systematic review. J Palliat Med 2012;15(2):228-35.

24. Almutary H, Douglas C, Bonner A. Multidimensional symptom clusters: an exploratory factor analysis in advanced chronic kidney disease. J Adv Nurs 2016;72(10):2389-400.

25. Gutiérrez D, Leiva-Santos JP, Macías MJ, Cuesta AI. Perfil sintomático de los pacientes con Enfermedad Renal Crónica Estadio 4 y 5. Enferm Nefrol 2017;20(3):259-66.

26. Kiecolt JK, McGuire L, Robles TF, Glaser R. Emotions, morbidity, and mortality: new perspectives from psychoneuroimmunology. Annu Rev Psychol 2002;53:83107.

27. Kimmel PL, Patel SS. Quality of life in patients with chronic kidney disease: focus on end-stage renal disease treated with hemodialysis. Semin Nephrol 2006, 26(1) 68-79. 
28. Lahey B. Public health significance of neuroticism. Am Psychol 2009;64(4):241-56.

29. Grossardt BR, Bower JH, Geda YG, Colligan RC, Rocca WA. Pessimistic, anxious, and depressive personality traits predict all cause mortality: the Mayo Clinic cohort study of personality and aging. Psychosom Med 2009;71(5):491-500.

30. Perales-Montilla CM, Duschek S, Reyes-Del Paso $\mathrm{GA}$. The influence of emotional factors on the report of somatic symptoms in patients on chronic hemodialysis: the importance of anxiety. Nefrología 2013;33(6):816-25.

31. Steinhauser KE, Christakis NA, Clipp EC, McNeilly M, McIntyre L, Tulsky JA. Factors considered important at the end of life by patients, family, physicians, and other care providers. JAMA 2000;284(19):2476-82.

32. Ocharán J. Cuidados paliativos en la enfermedad renal crónica. Gaceta Médica de Bilbao 2016;2112(4):209-15.
33. Axelsson L, Alvariza A, Lindberg J, Öhlén J, Håkanson C, et al. Unmet Palliative Care Needs Among Patients With End-Stage Kidney Disease: A National Registry Study About the Last Week of Life. Journal of Pain and Symptom Management 2018;55(2):236-44.

34. Bayés R, Limonero JT, Barreto P, Comas MD. A way to screen for suffering in palliative care. J Palliat Care 1997;13(2):22-6.

35. Llantá MC, Pire T, Grau J, Vilaú L, Massip C, et al. Evaluación del sufrimiento en pacientes con quimioterapia del Instituto Nacional de Oncología y Radiobiología. Psicología y Salud 2008;8(2):149-54.

36. Bruera E, Kuen N, Miler MJ, Selmser P, MacmiIlan K. The Edmonton Sympton Assesment System (ESAS): a simple method for the assessment of paIliative care patients. J Palliat Care 1991;7:6-9.

Este artículo se distribuye bajo una Licencia Creative Commons Atribución-NoComercial 4.0 Internacional. https://creativecommons.org/licenses/by-nc/4.0/

Open Access (C) () () 